\title{
THE EXTRINSIC NOISE EFFECT ON LATERAL INHIBITION DIFFERENTIATION WAVES
}

\author{
ANDREAS I. REPPAS ${ }^{1}$, GEORGIOS LOLAS ${ }^{1}$, ANDREAS DEUTSCH ${ }^{2}$ AND HARALAMPOS HATZIKIROU ${ }^{1}$
}

\begin{abstract}
Multipotent differentiation, where cells adopt one of several cell fates, is a determinate and orchestrated procedure that often incorporates stochastic mechanisms in order to diversify cell types. How these stochastic phenomena interact to govern cell fate are poorly understood. Nonetheless, cell fate decision making procedure is mainly regulated through the activation of differentiation waves and associated signaling pathways. In the current work, we focus on the Notch/Delta signaling pathway which is not only known to trigger such waves but also is used to achieve the principle of lateral inhibition, i.e. a competition for exclusive fates through cross-signaling between neighboring cells. Such a process ensures unambiguous stochastic decisions influenced by intrinsic noise sources, e.g. as ones found in the regulation of signaling pathways, and extrinsic stochastic fluctuations, attributed to micro-environmental factors. However, the effect of intrinsic and extrinsic noise on cell fate determination is an open problem. Our goal is to elucidate how the induction of extrinsic noise affects cell fate specification in a lateral inhibition mechanism. Using a stochastic Cellular Automaton with continuous state space, we show that extrinsic noise results in the emergence of steady-state furrow patterns of cells in a "frustrated/transient" phenotypic state.
\end{abstract}

\section{INTRODUCTION}

Cell fate determination during developmental processes requires the integration of lineage information and signaling cues at specific developmental time points to yield robust, reproducible cell fate executions. Most cell fate decisions are determinate and are typically coordinated by waves of differentiation. Such "waves of fate" have been observed both during the development of the Drosophila visual system [44] as well as in the vertebrate retina [9, 25]. More specifically, for the Drosophila visual system differentiation waves are formed in different parts of the embryo following specific morphogenetic furrows in order to establish topographic connections throughout the fly visual system. Additionally, in retinal development, retinal progenitor cells undergo a series of state changes before they adopt a final differentiated fate while a state is defined by the cell competence to respond to both intrinsic and extrinsic cues. The synchronization of these multiple differentiation waves is mediated by specific signaling pathways. One of the most important signaling pathways is the Notch/Delta [6, 10, 22, 25, 30, 41].

The Notch/Delta pathway represents a juxtacrine signaling transduction mechanism in developmental biology for cell fate decisions, in particular in the nervous system [2,32. It is also known as the "lateral inhibition" or the "lateral specification" mechanism, where the selected cell blocks the ability of its neighbours to differentiate. More specifically, in lateral cell fate specification neighboring cells exchange signals in order to adopt a specific fate, while one "local" winner is selected. In this regard, in small domains a single "winner" is produced whereas in larger fields a self-organizing "salt-pepper" or chessboard pattern is formed [2].

The main factors of the Notch/Delta pathway involve the extracellular domain of the two transmembrane ligands Delta and Serrate on the surface of one cell interacting with the extracellular domain of Notch receptor at the adjacent cell. The ligand/receptor binding triggers proteolytic events that result in the cleavage of the receptor's intracellular domain. Notch interacts with Delta in two modes: activating Delta signaling in neighboring cells (trans-activation) while inhibiting Delta

1 Center for Advancing Electronics Dresden, Technische Universität Dresden, Germany, ${ }^{2}$ Center for Information Services and High Performance Computing, Technische Universität Dresden, Germany 


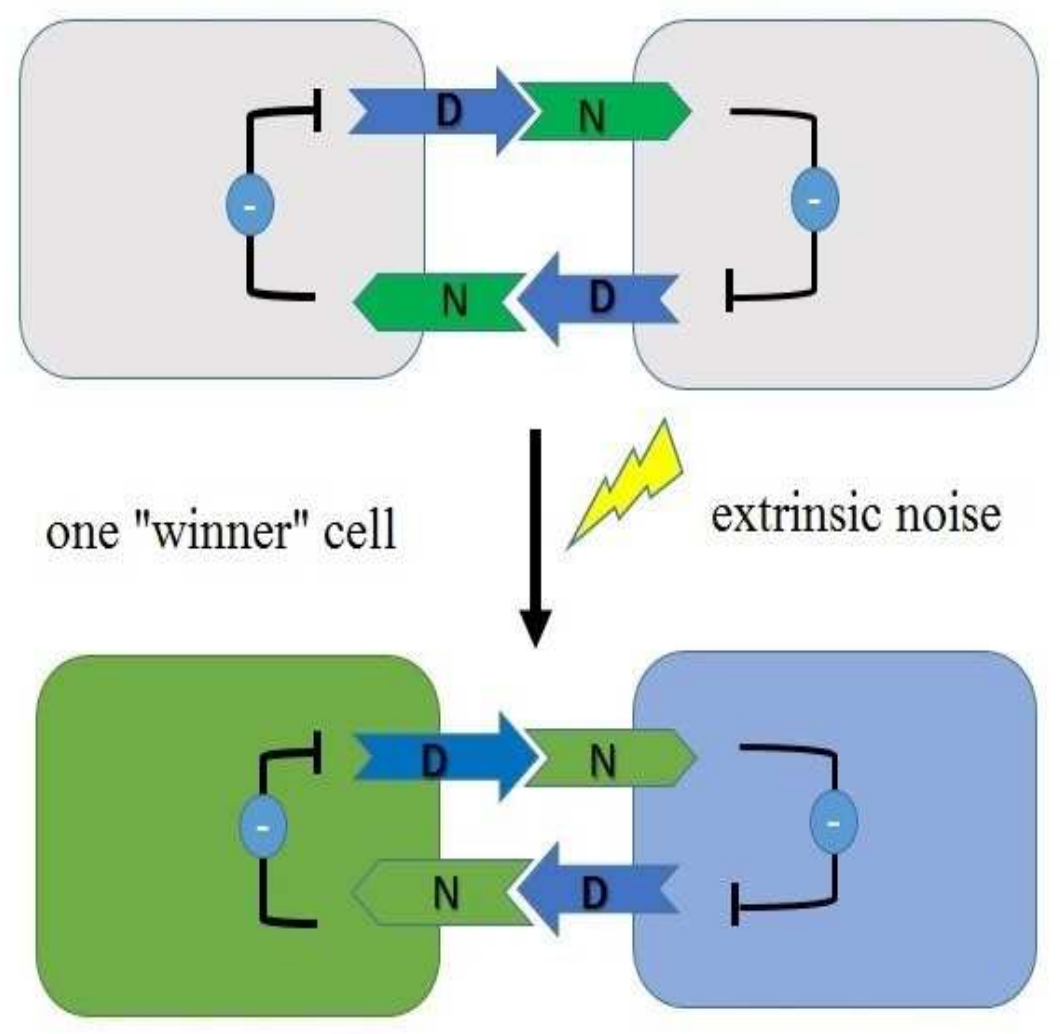

FiguRE 1. Notch/Delta mechanism: The Notch receptor is activated upon binding to the Delta ligand that is anchored to the membrane of an adjacent cell. The combination of intercellular Notch activation and intracellular Delta deactivation results in two mutually exclusive fates of initially equipotent neighboring cells.

signaling in the same cell (cis-inhibition). Lateral inhibition patterning can emerge when Notch signaling downregulates Delta levels. Under specific conditions a high level of Notch in one cell will drive all of its neighbors to low levels of Notch and vise versa in the Delta case. This generates a stable lateral inhibition pattern where each over-expressing Notch cell is surrounded by cells with increased Delta concentration (see Figure 1).

Although most of cell fate decisions are fixed within the developmental process, not all of them are determinate. Some cell fate decisions require random selection among alternatives. In agreement with determinate decisions, mechanisms acting after stochastic choices can compensate to yield robust outcomes [27, 31]. Two stochastic influences can play crucial roles: the external and the internal noise. The intrinsic noise is associated with stochasticity involved in the genetic, epigenetic, transcriptional or translation regulations. On the other hand, extrinsic noise accounts for all the microenvironmental factors such as extracellular matrix or stromal components that can influence the cell fate determination process. In this work, we investigate the impact of extrinsic noise on the robustness of pattern formation introduced by the Notch/Delta mechanism. Here, the extrinsic noise coarse-grains the factors impacting Notch/Delta binding at the cells' membrane. We show that extrinsic noise can result in the formation of spatio-temporal "frustrated furrows" associating our results with recently discovered biological evidence. Although the word noise is related with negative effects as something undesirable, in biological systems noise represents "randomness". In other words, noise may well be regarded as something desirable.

The structure of this paper is as follows: In section 2, a thorough investigation of recently developed theoretical models of lateral inhibition is presented while in section 2.1 a simplified "lateral inhibition" model of Collier et al. [1996] is defined. The patterning bifurcation diagram in a two-cell population is demonstrated in section 3.1. The fine grained "salt and pepper" pattern formation 
structure is presented and analyzed in subsection 3.1.1. A stochastic differential equation formulation for Notch-Delta is then derived in section 3.2. In subsection 3.2.1, our simulations results highlighting the emergence of a "frustrated" cell state associated with the effect of extrinsic noise are analyzed and compared with the deterministic case. In section 3.3 we investigate the preponderance of "frustrated/transient" cell fate mechanism in biological systems. A discussion together with suggestions for future work is finally given in section 4 .

\section{Modeling Lateral Specification}

Several mathematical models have been developed to analyze lateral specification in different biological contexts. The first model on juxtacrine signalling was formulated by Collier et al. [1996] considering the role of a ligand, Delta, and its receptor, Notch, and demonstrating that lateral inhibition is able to generate fine-grained patterns. There have been other extended formulations of the aforementioned model, by Owen and coworkers, either by considering the dynamics of ligands as well as free and bound receptors [49] or by inducing positive feedback realizations for both ligand and receptor [40,48], applied to different geometries (strings of cells and square and hexagonal arrays), and proving that lateral inhibition can generate patterns with longer wavelength. In a more recent paper, O'Dea and King [2011] developed a multi-scale technique in order to construct a continuum model for investigating the pattern formation dynamics proposed in Collier et al. [1996].

Models dealing mostly with genetic circuits through the nonlinear lateral inhibition mechanism have been proposed by Plahte [2001] and Shaya and Sprinzak [2011]. More detailed models of the Notch/Delta circuit can be found in Hsu et al. [2006] and Meir at al. [2002]. More specifically, Hsu et al. [2006] demonstrated that the lateral inhibition mechanism favors cells that are in contact with fewer inhibitory signal sending adjacent cells while Meir at al. [2002] investigated the parameter regime and how this affect the generation of new patterns through the lateral inhibition mechanism.

Just recently, several quantitative models have been developed in order to address specific experimental questions associated with the specific pathway [12,29, 35]. Koizumi et al. [2012] studied the role of lateral inhibitory regulation on cells present at the leading edge (tip-cells) during Drosophila trachea development. Both their mathematical and numerical results revealed that Notch/Delta mechanism enhances the robustness of the tip-cell selection compared with a system regulated by self inhibition. The work by Cohen and coworkers [12, 35] showed that the development of the microchaete bristle pattern on the notum of the fruitfly Drosophila melanogaster is dependent upon a long-range interacting population mediated by dynamically extending actin-based filopodia. Cells use filopodia-like extensions to gather information from non-neighboring cells. Non-local cell interactions mediated by filopodia dynamics are thought to help cells receive morphogen signals as well as collect information regarding the identity of nearby cells that provide survival cues. These filopodia dynamics generate a type of structured noise that contributes to the formation of a well-ordered and spaced pattern of bristles by the induction of intermittent Notch-Delta signaling.

An investigation of the role of structural noise (spatial as well as temporal) was presented in a more recent work of Cohen et al. [11. They proposed an asynchronous cellular automaton model to study a lateral inhibition mechanism in a hexagonal lattice. They showed that in the absence of noise they were able to recapitulate the results obtained from previous related continuous models using a discrete formulation without the need to invoke diffusible morphogens. Whereas the inclusion of noise can lead to stripped or spotted pattern refinement whose thickness is directly dependent on the noise signal threshold [11.

Additionally, Sprinzak et al. [47] have studied Notch signaling from a synthetic biology perspective. In particular, they highlighted the issue of cis-inhibition effect, namely what happens if Notch and Delta bind in the same cell alongside with the trans-inhibition effect. In their seminal work, they developed different kinetic models that could reproduce the dynamic behavior of the engineered Notch-Delta circuits. Mainly, their models were reduced to three equations in which the dynamics between the ligand, the receptor and Notch intracellular domain are investigated.

It has recently been observed by Formosa and Ibanes [21] that cis-inhibition enriches cell patterning by promoting pattern multistability. More specifically, their work predicted novel characteristics of 
the Notch-Delta pathway such as the role of ligands that travel through the extracellular space and the role of diffusible morphogens on patterns wavefronts. Moreover, they showed the antagonistic role of Notch acting either as a lateral inducer or lateral inhibitor in specific developmental processes. For instance, they predicted that autoactivation of a specific proneural factor drives Delta upstream and is a fundamental component for chicken inner ear robustness [42]

A recent work by Barad and co-workers highlighted that the ability of Delta to inhibit Notch within the same cell (cis-interaction) can reduce the sources of errors associated with the lateral inhibition process [3,4]. They argue that this beneficial effect of noise complements its role in the generation of phenotypic diversity.

Several elements of the Notch/Delta pathway remain unclear. For example, the diffusible transport of ligands through the extracellular space suggest a new way of non-local cell-cell communication. Another example is the "bipolar" role of Notch/Delta. Namely, it is known that Notch can have both an inductive as well as an inhibitory role in specific organs. A thorough review of the aforementioned models as well as the latest scientific challenges in pattern formation through the Notch-Delta pathway can be found at [20].

2.1. Mathematical Model of Lateral Specification. In the current work, we construct a model based upon the mathematical framework proposed and analysed by Collier et al. [1996]. The model is defined by a set of coupled ordinary differential equations that describe the dynamics of signaling mediated Notch-Delta intercellular activation and intracellular inhibition. A pair of ODE's governs the evolution of the levels of Notch and Delta activity in each cell $i$ over time $\tau$. The Collier et al. [1996] model in dimensionless form is given by:

$$
\begin{gathered}
\dot{n}_{i}=f(\bar{D})-n_{i}, \\
\dot{d}_{i}=g\left(n_{i}\right)-d_{i},
\end{gathered}
$$

where $\bar{D}$ denotes the mean level of Delta activity in the cells surrounding cell $i, \bar{D}=\frac{1}{\left|\mathfrak{N}_{i}\right|} \sum_{j \in \mathfrak{N}_{i}} D_{j}$, where $\mathfrak{N}_{i}$ denotes the neighbourhood of cell $i$ and $\left|\mathfrak{N}_{i}\right|$ specifies the number of its neighbours. To model the lateral inhibition exerted by one cell upon another, Collier et al. [1996] defined the functions $f, g$ as:

$$
\begin{aligned}
& f(x)=\frac{x^{k}}{\bar{\alpha}+x^{k}}, \\
& g(x)=\frac{1}{1+\beta x^{h}},
\end{aligned}
$$

where $f$ is a monotonically increasing function modeling the coupling between adjacent cells and $g$ is a monotonically decreasing function representing the inhibitory effect of the bound Notch/Delta upon Delta production. The positive parameters $\bar{\alpha}, \beta, k, h$ determine the feedback strength. More specifically, the value of the exponent $k$ determines whether the Notch-Delta binding is monovalent $(k \geq 1)$ or cooperative $(k \geq 2)$. Cooperative reaction means that the Notch receptor has more than one binding cites, so the receptor has the ability to bind to more than one ligand molecules while in the monovalent case $(k=1)$ the Notch receptor has only one binding site. Here, we assume the monovalent case. In this regard, without loss of generality, we approximate function $f=a x$, noting that this latter formulation coincides with the Taylor expansion of equation (2a) for $x \ll 1$ and $\alpha=\bar{\alpha}^{-1}$. The reduced model is then given by:

$$
\begin{array}{r}
\quad \dot{n}_{i}=\alpha \bar{D}-n_{i}, \\
\dot{d}_{i}=\frac{1}{1+\beta n_{i}^{h}}-d_{i} .
\end{array}
$$


Parameter $\alpha$ in equation (3a) depicts the normalized strength of interaction between neighboring cells. Since no explicit discussion of the biological motivation of the parameter estimation chosen by Collier et al. [1996] was presented, we set the parameters:

$$
h=5, \beta=100
$$

By using a linear response of Notch production we seek to investigate the immediate effect of Delta concentration on the production of Notch activity (in the neighboring cells) by just tuning parameter $\alpha$ which served as our bifurcation parameter. Below, we will investigate the dynamics of pattern formation.

\section{Results}

In this section, we present the results of our analysis for a deterministic and a stochastic version of the model.

\subsection{Bifurcation analysis of the Deterministic case in the absence of External noise.} Firstly, we determine under which parameter regimes "salt and pepper" patterning is achieved with neighboring cells adopting opposing fates. Since the Notch/Delta mechanism under consideration is local, analyzing the model equations for two cells with periodic boundary conditions gives insight into the period-two patterning behavior. Figure 2 presents the bifurcation diagrams of the Notch and Delta activation computed as fixed points of Equations $3 \mathrm{a}$, 3b with respect to the parameter $\alpha$. The bifurcation diagrams were extracted by performing an arc-length continuation method [28]. The existence of a pitchfork bifurcation for a critical value of $\alpha$ leads to a bistable regime of Notch and Delta dynamics associated with a saddle point.

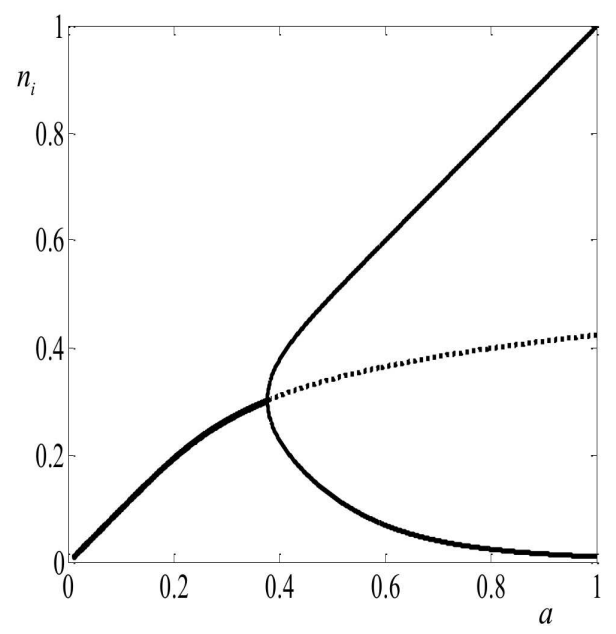

(a)

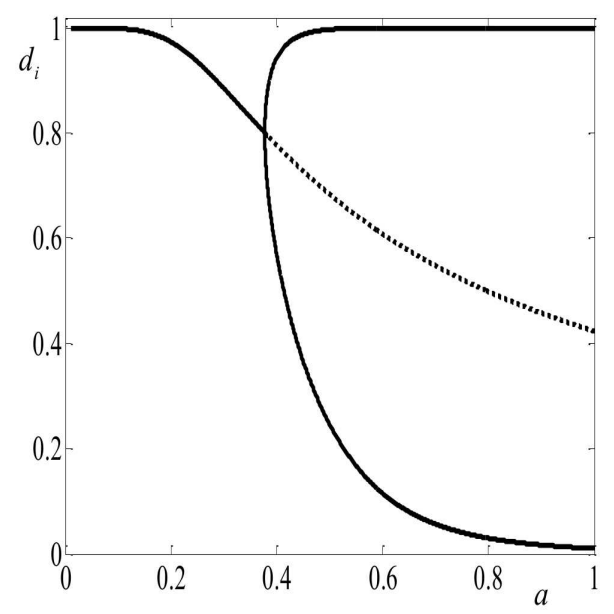

(b)

FiguRE 2. Bifurcation diagram: Bifurcation diagram of the Notch concentration with respect to the strength of interaction, $\alpha$, in a pair of cells. The two stable solution curves (solid lines) in the central region correspond to the period-two fine-grained pattern, while the unstable state corresponds to the homogeneous state (dotted lines). The critical point which initiates pattern formation is $\alpha_{c r}=0.3771$.

It can be seen, that if the strength of interaction is sufficiently small the only stable solution is the homogeneous state where all the cells have the same concentration of Notch and Delta. At a critical value, $\alpha_{c r}$, the homogeneous state loses stability and gives rise to a pair of heterogeneous steady states where the cells adopt two distinct fates: high-Notch/low-Delta (primary fate) and lowNotch/high-Delta (secondary fate). Thus, when $\alpha>\alpha_{c r}$ the two-cells system results in a mutual inactivation which creates the lateral inhibition pattern. 
3.1.1. Numerical simulations. We consider a coupled map lattice model with continuous state defined on a two-dimensional regular lattice $L=L_{1} \times L_{2}$, where $L_{1}=L_{2}=\{1, \ldots 50\}$ are the lattice dimensions, with 2500 nodes (cells) interacting in a von Neumann neighborhood with periodic boundary conditions. The state space of the model is $\left(n_{i}, d_{i}\right) \in[0,1]^{2} \subset \Re^{2}$. In order to study the fate specification wave we initialize our system by assuming a line of cells with opposite fates (inhibiting one another) while the rest of the cells are set to a neutral state (no fate state), in other words having zero concentration of Notch and Delta (Figure 3(a)). The aforementioned model can be viewed as a Cellular Automaton with continuous state space coupled with synchronous update rules with the lateral inhibition process described by equations $1 \mathrm{a}$ and $1 \mathrm{~b}$. In other words, the transition rules associated with lateral inhibition can be represented by the following simple mechanism: the level of Notch activation, $n$, reflects the intensity of the inhibition the cell experiences while the level of Delta activity, $d$, reflects the intensity of the inhibitory signal that each cell delivers to its neighbors. More specifically, a cell surrounded by neighboring cells expressing high Notch is assumed to be in an inactive state (state 0), while a cell surrounded by neighboring cells expressing low Notch is assumed to be in an active state (state 1) In each case we performed simulation for parameter values $\alpha>\alpha_{c r}$ aiming to investigate how the fate specification wave propagates in space and time.

We solve the system of coupled equations $3 \mathrm{a}$, 3b numerically using a Runge-Kutta Method of $4^{\text {th }}$ order for each of the $N=2500$ cells. Figure 3 presents the evolution of the fate wave specification when $\alpha=1$. As expected, the initial line of fate specific cells evolves through time creating a fate wave that results in the evolution of a "salt and pepper" pattern.

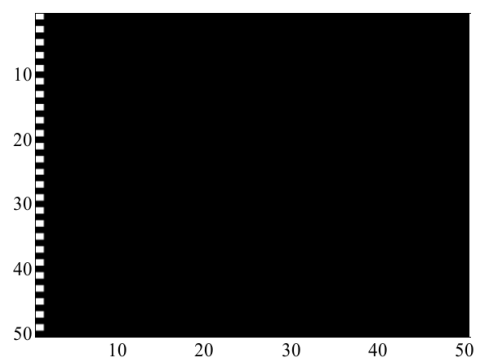

(a)

$\tau=0$

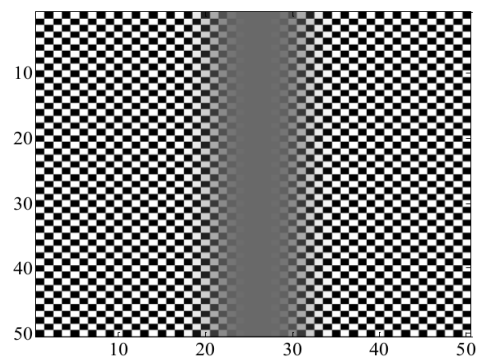

(c)

$\tau=30$

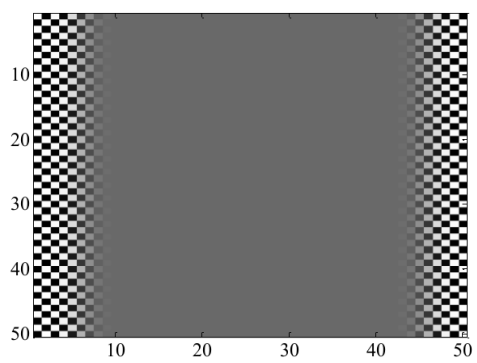

(b)

$\tau=20$

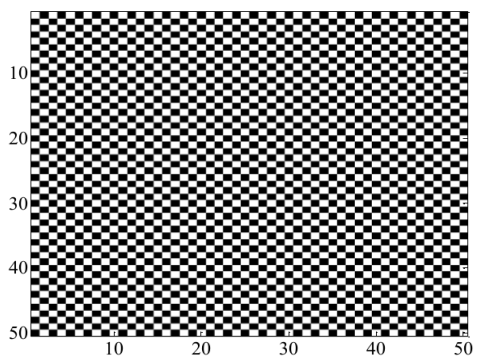

(d)

$\tau=40$
Low Notch

$\square$ High Notch

FiguRE 3. Deterministic fate specification wave: The generation of a "finegrained" chessboard pattern in simulations of the deterministic model defined by the system of equations (3a) and (3b) with $\alpha=1$.

In figure 4(a) we investigate the evolution of Notch concentration between two different cells. Spatial patterning is generated by a wave front, behind which a regular "salt and pepper" pattern forms. Initially, the level of Notch concentration in all cells (except the first line with cells having already decided their fate) is chosen close to the stable manifold of the saddle point (see Figure 2). Figure 4(b) depicts the phase space of the Notch concentration between two consecutive pairs of cells 


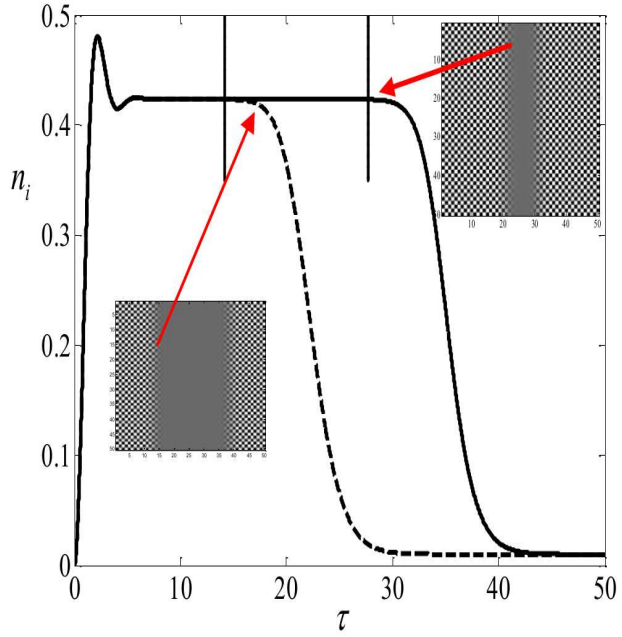

(a)

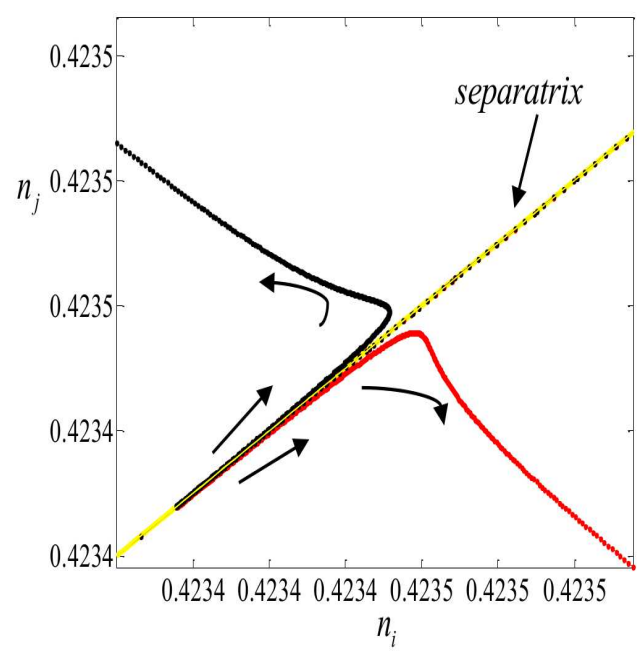

(b)

FigURE 4. Impact of differentiation on cell dynamics: (a) Time evolution of the Notch concentration of different cells. The vertical lines depict the time of fate selection for each cell. (b) Phase space of Notch concentrations between different cells. The trajectories correspond to consecutive neighboring couples of cells which adopt different fates. The yellow line is the stable manifold of the saddle node (see text for further information).

which finally adopt different fates. The stable manifold of the saddle point (denoted in yellow in the plane $\left(n_{i}, n_{j}\right)$ in Figure 4(b)) separates the attraction of the two different states [38]. The cells remain on the stable manifold until the wave fate specification forces them to follow the steady-state pattern.

3.2. Stochastic case: The emergence of the third "frustrated" state as a consequence of external noise. Here, we investigate the impact of extrinsic noise on the pattern formation dynamics. We assume that extrinsic noise influences the cell's ability in receiving free Delta $\bar{D}$ and that the parameter $\alpha$ is a random variable $\alpha=\mu+\eta_{\tau}$, with $\mu \in[0,1]$ and $\eta_{\tau} \sim \mathcal{N}\left(0, \sigma^{2}\right)$. The rest of the parameters are defined by equation (4) presented in section 2. Thus, the stochastic version of the deterministic model defined in equations (3a, 3b) is:

$$
\begin{array}{r}
\dot{n}_{i}=\mu \bar{D}-n_{i}+\bar{D} \eta_{\tau} \\
\dot{d}_{i}=\frac{1}{1+\beta n_{i}^{h}}-d_{i}
\end{array}
$$

3.2.1. Numerical simulations. Following the simulation setting presented in section 3.2 , we simulate the evolution of the fate specification wave by initializing the system as in the deterministic case. For the numerical integration of equations (5a) and (5b) in each cell $i$ we used the Euler-Maruyama method with time step $d \tau=0.01$ [33].

As expected, the extrinsic noise perturbs the steady state "salt and pepper" pattern found in the deterministic case. In this regard, in Figure 5, at $\tau=20$ we note that cells in the middle of the domain select a fate before the arrival of the differentiation wave. As time evolves in $\tau=40$, we observe that most of the cells have already been differentiated. However, several closed paths of "frustrated/transient cells", i.e. undecided ones, emerge enclosing "chessboard" patterned cells. By time $\tau=100$, a combination of a fine grained pattern of "salt and pepper" and "furrows of frustrated cells" has emerged. 


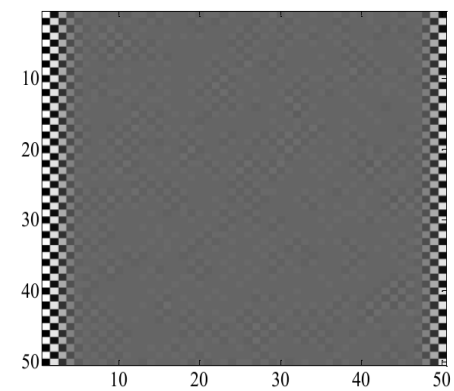

(a)

$\tau=10$

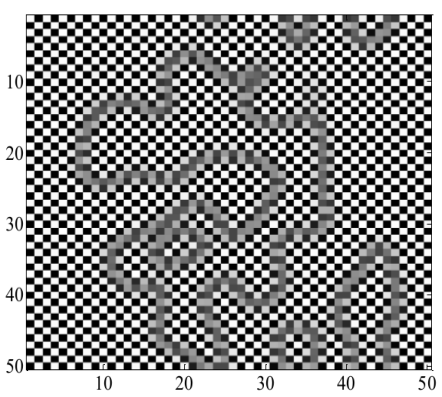

(c)

$\tau=40$

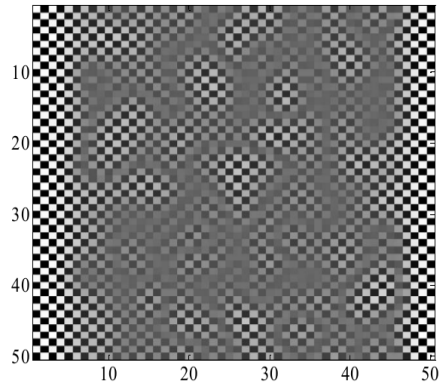

Low Notch

High Notch

Frustrated state (b)

$\tau=20$

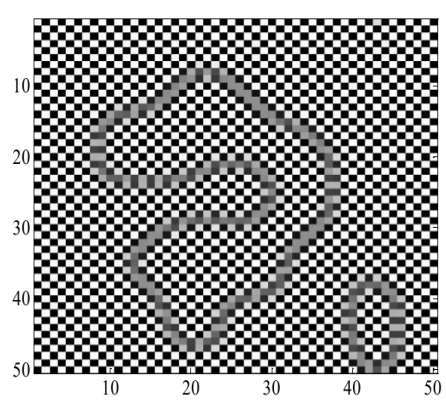

(d)

FigURE 5. Emergence of frustrated states: The evolution of emerging "frustrated furrows" within the "salt and pepper" pattern in simulations of the stochastic system of equations (5a, 5b), with parameters $\mu=1$ and $\sigma=0.01$.

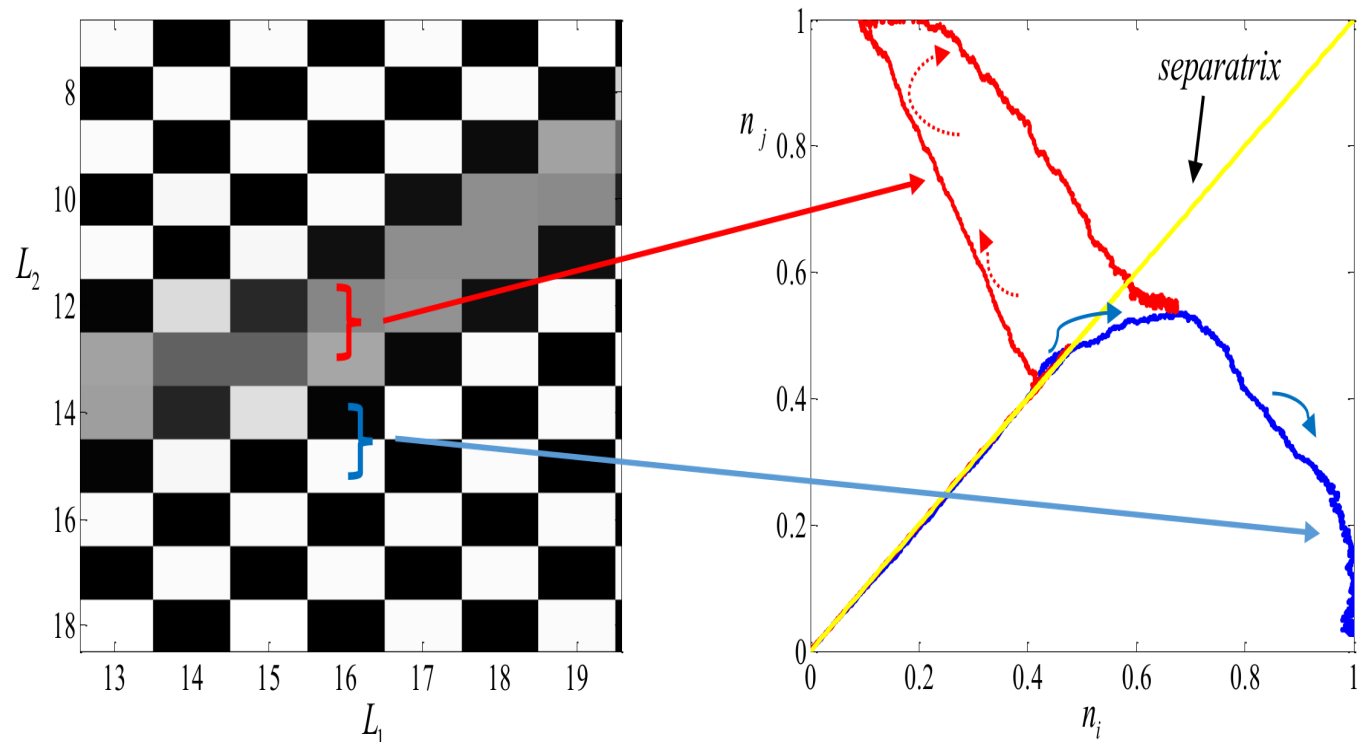

(a)

(b)

FiguRE 6. "Frustrated furrows" dynamics: (a) A close look to a neighborhood of a "frustrated furrow" reveals cell fate switching. (b) Phase portrait of Notch concentrations between different couples of cells. See text for further details.

By having a closer look at Figure 6(a), we see that these "frustrated furrows" are formed by cells that have adopted a fate which is different from the deterministic case. The exact formation of these 
furrows is varying for different system realizations. As we can observe in Figure 6(a), as soon as the differentiation wave has reached these frustrating furrows a conflict in cell fate determination arises: cells in the region within the "frustrated furrows" have opposite order of successive black and white nodes compared to cells in the region outside the furrows. Cells located at the vicinity of these two regions remain undecided, in terms of their fate specification, and therefore the frustrated/transient or mixed state perpetuates in time (frustrated cells denoted by gray in Figure 6(a)).

Additionally, in Figure 6(b) we highlight the Notch concentration of two neighboring cell pairs. In one pair (blue curve) noise forces the trajectory to cross the stable manifold, implying the selection of opposite cell fates compared to the deterministic case as shown in Figure 4(b) while the other pair of cells (red curve) stay close to the separatrix. Furthermore, in the stochastic model "fate selection" takes place earlier than in the deterministic case (Figure 7).

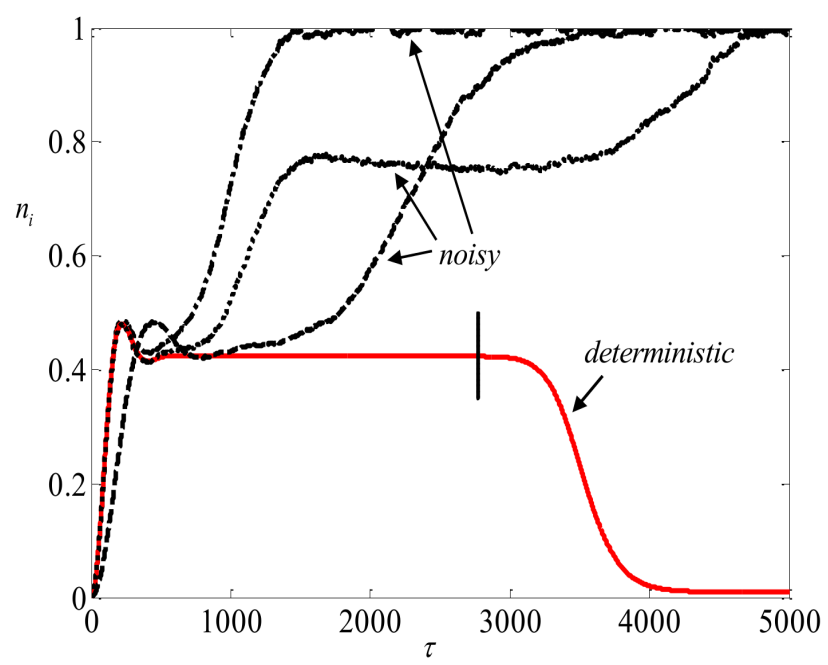

FiguRE 7. Effect of noise on cell fate specification: Comparison of a cell's Notch level time evolution in the presence of noise $(\mu=1$ and $\sigma=0.01)$ and in the deterministic case $(\alpha=\mu=1$ and $\sigma=0)$. Stochastic fluctuations can induce cell fate selection before the arrival of the differentiation wave. The vertical line illustrates the time of cell fate decision in the deterministic case.

Finally, in Figure 8(a) and 8(c) we compare the state distribution in the deterministic $(\mu=1$ and $\sigma=0)$ and the stochastic case $(\mu=1$ and $\sigma=0.01)$, averaging over 100 simulations for time $\tau=100$. The intermediate states denote the existence of frustrated cells found in the "fate switching furrows". Additionally, in Figure 8(b) and 8(d), we compare the Notch dynamics in a cross-section of the lattice for both cases. As expected, in the deterministic case half of the cells adopt the high Notch fate while the rest adopt the low Notch one. On the contrary, in the stochastic case, the emergence of frustrated cell furrows divides the domain into patterned regions.

We also studied the effect of varying the noise amplitude. A significant range of noise levels gave rise to emerging frustrated regions within the "salt and pepper" pattern. By increasing the level of noise beyond $\sigma>0.3$ we obtained a fully disordered system (Figure 9). Additionally, we investigated the effect of neighborhood topology, i.e. the signaling communication range. For a Moore neighborhood (8 immediate neighbors) a refined stripped pattern is obtained (Figure 10(a)). In a similar manner, when noise is implemented frustrated furrows emerge within the stripped pattern, as shown in Figure 10(b). The aforementioned results are in line with those obtained by Cohen et al. [2010] using a hexagonal lattice. In the same study, the authors suggested that Notch/Delta spatiotemporal dynamics are similar to phenomena found in solid state physics, such as grain growth and recrystallization [36, 37]. Adding to this discussion point, our results suggest that dynamics of the frustrated patterns are similar with spin glasses phenomena. In the next section, we discuss 
the biological relevance of the Notch/Delta mechanism and the existence of frustrated/transient phenotypic cells states.

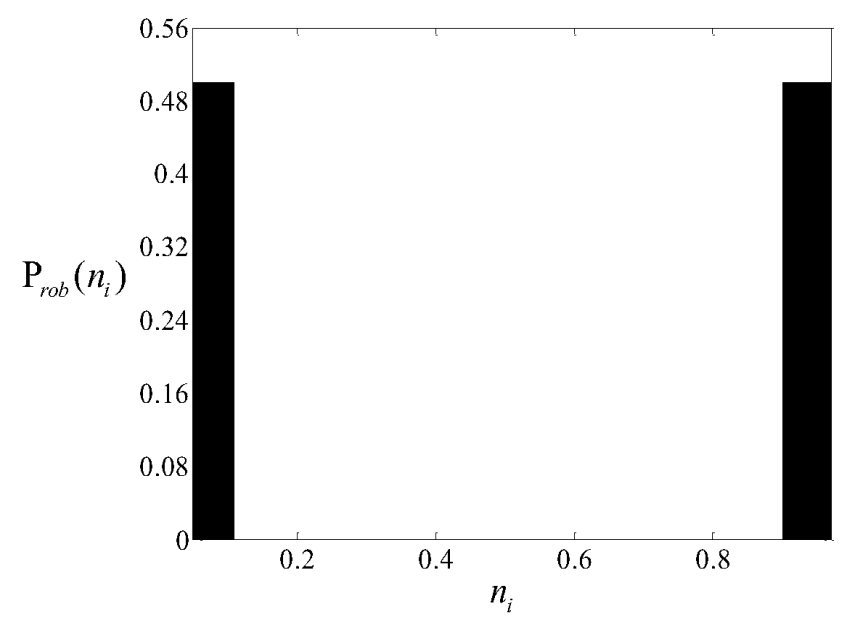

(a)

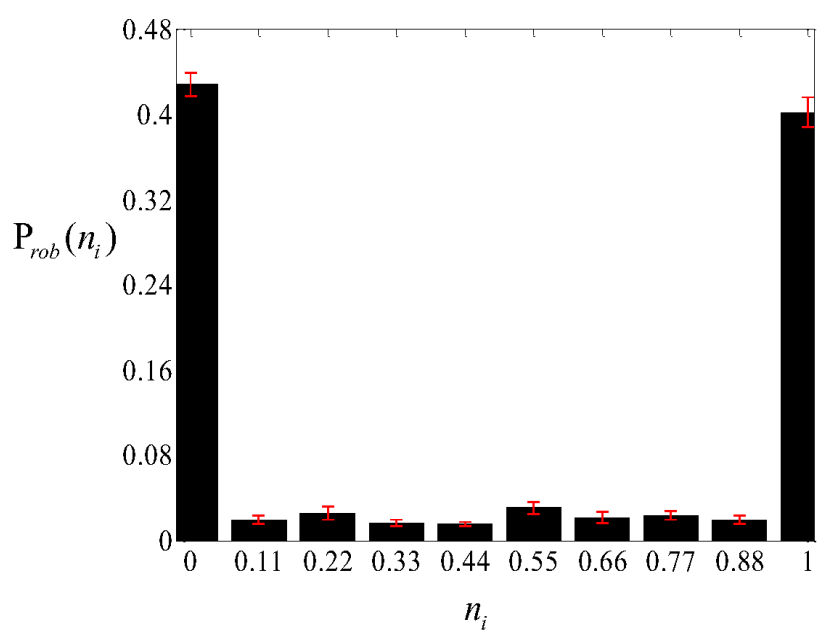

(c)

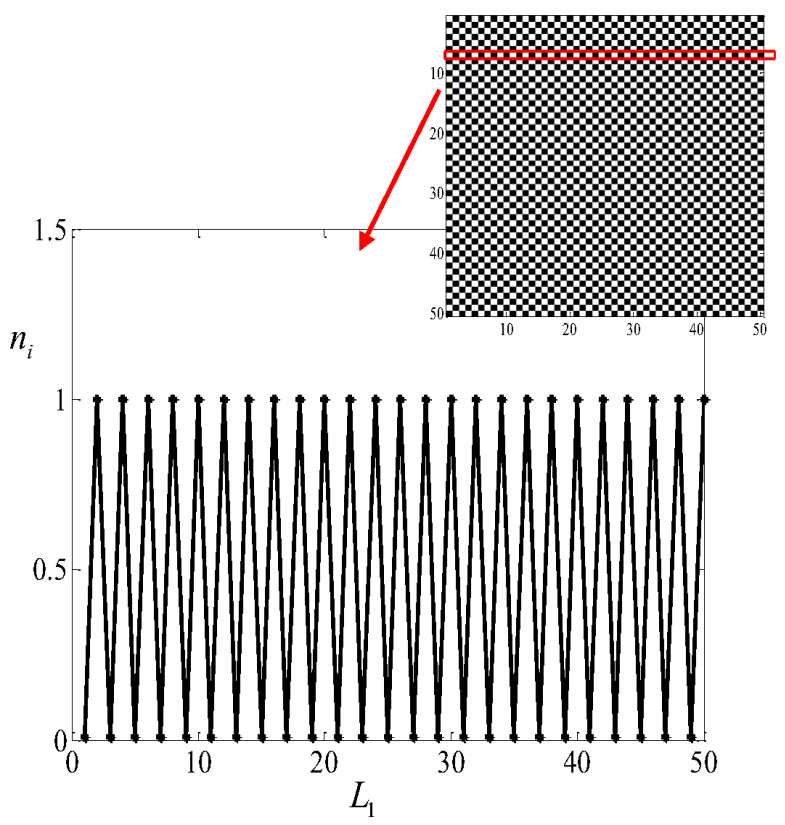

(b)

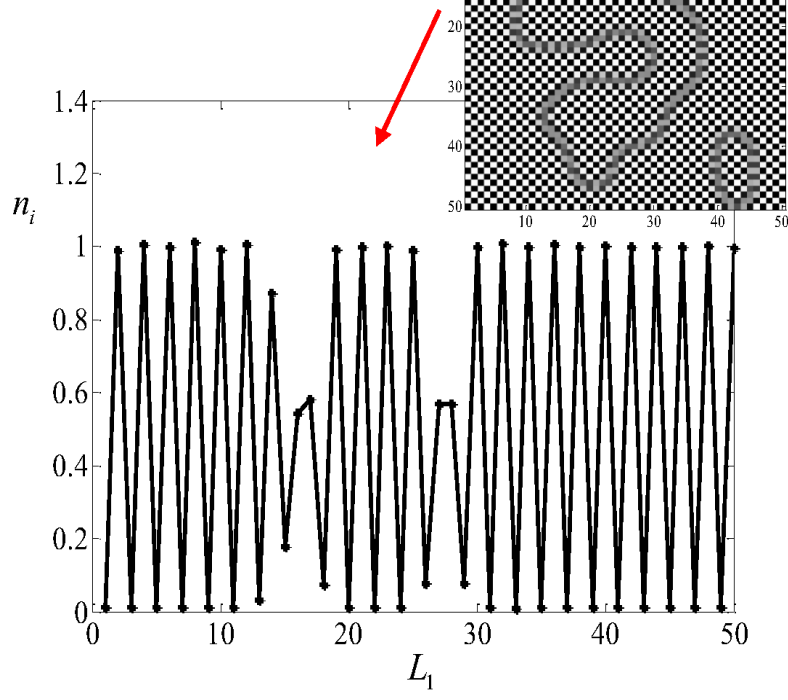

(d)

FiguRE 8. Notch probability distribution: (a) Notch probability distribution in the deterministic case, when $\alpha=\mu=1$. Half of the cells adopt the high Notch fate and the other half the low one. (b) Notch level in one-dimensional cross sections for the deterministic case. (c) Notch probability distribution in the stochastic case for $\mu=1$ and $\sigma=0.01$ averaged over 100 simulations for time $\tau=100$. Some cells adopt an intermediate state. (d) Notch level in one-dimensional cross sections for the stochastic case.

3.3. The frustrated/mixed state in cell-fate decision making. On the basis of the above considerations, we have raised the possibility that during development cell decision making processes do not follow a deterministic chain of events in adopting their final fate. Instead, we propose that 


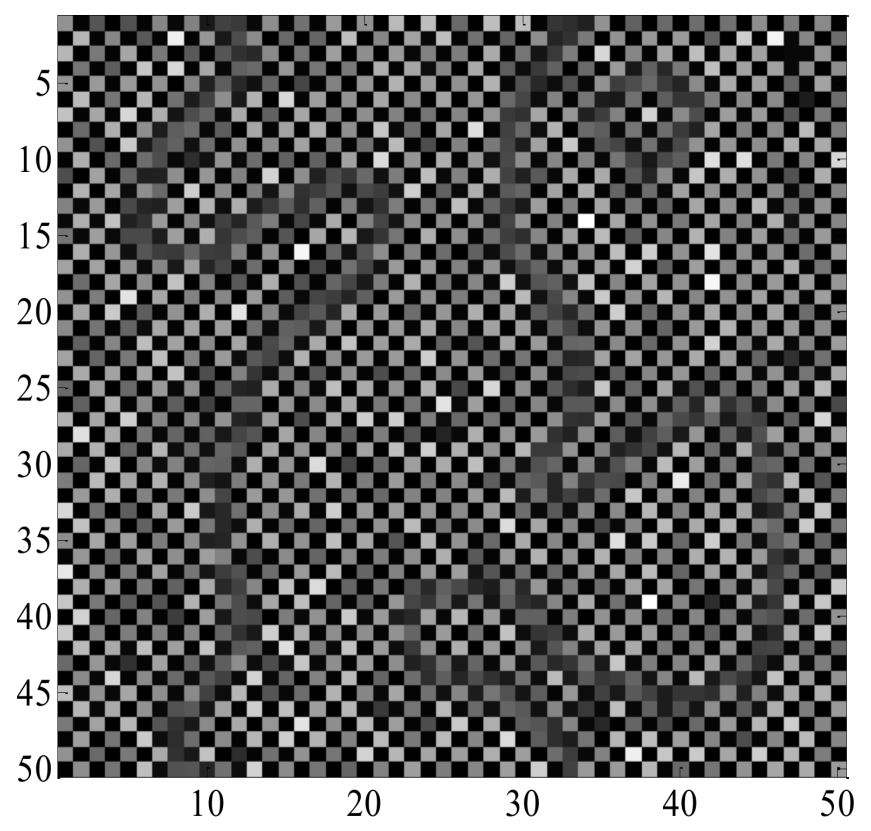

FiguRE 9. Disorder for high intensity noise: Simulations of the stochastic system of equations (5a, 5b), with parameters $\mu=1$ and $\sigma=0.35$. Furrows of cells in a "frustrated" /transient state evolve through the system resulting in a fully disordered pattern.

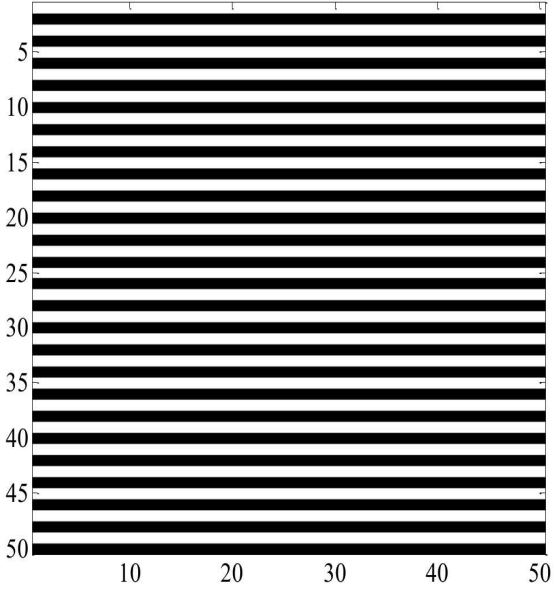

(a)

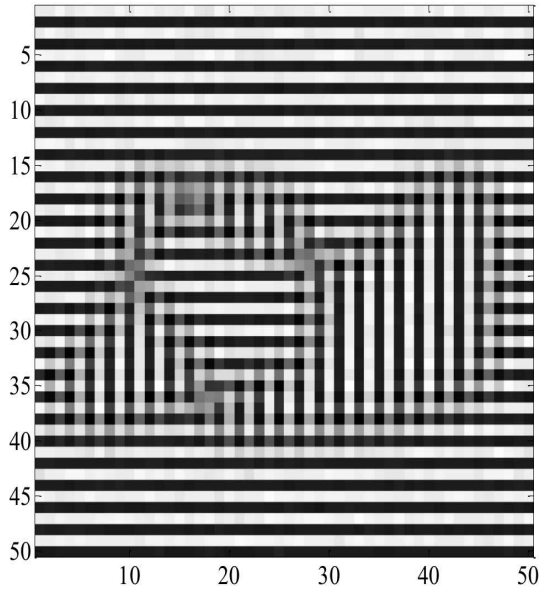

(b)

FiguRE 10. Neighborhood effect on the Notch/Delta model:(a) A pattern of consecutive high Notch (low Delta) and low Notch (high Delta) stripes is obtained in the case we assume interactions with 8 neighbors for each cell. (b) The emergence of frustrated furrows in simulations of the stochastic system of equations (5a, 5b), with parameters $\mu=1$ and $\sigma=0.02$ when each cell interacts with 8 neighbors.

extrinsic noise in cell fate specification signaling may force cellular sub-population to a metastable, transient, frustrated state. Even though many of the components regarding differentiation programs are known, how noise may affect cell fate specification over time remains poorly understood. In this regard, it is not known if stochastic fluctuations are required before the decision point or after the decision has been made. 
Although a role for Notch/Delta signaling pathway as a lateral inhibitory mechanism is well established, it has been a subject to considerable probing of many other of its attributes in retinal development. In this regard, apart from its well known role as a cell fate mechanism, Cepko and coworkers [25, 26] proved recently that it could also be recruited to allow a preservation of a pool of retinal progenitor cells(RPCs) in a transient/frustrated competent state for later cellular diversification. During this competent/transient state, cells interact with both inhibitory and stimulatory environmental (extrinsic) factors to reach a fate decision. In a similar manner, Shin et al. [46] investigated the role of Notch signaling in regulating a motoneuron-interneuron fate decision. They proposed that transient Notch inactivation disrupts lateral inhibition, committing more precursors to lineages that produce ventral spinal cords (also known as pMNs) and interneuron cells. Whereas continuous inhibition of Notch activity produces excess pMNs and a deficit of interneurons.

The notion of mixed cell fate decision echoes recent experimental studies of the cellular mechanisms underlying retinal epiphysis. Cau and coworkers [7,8] revealed a new functional role for the NotchDelta pathway apart form its seminal role as the binary fate switcher. Namely, they proposed that Notch activation is required in order to seggregate epiphysial neurons that are in a transient phase of "double" or "mixed" identity. More specifically, in epiphysis there are two main type of cells; projection neurons and photoreceptors. However, a new type of cells has been identified of mixed identity. These cells have not yet received the postulated photoreceptor inducing signal while they have also not yet downregulated the projection neuron signal. Therefore, they remain in a mixed identity state. However, these mixed identity cells are rarely observed in wild-type epiphysis. Thus, it is speculated that either those cells do not represent a true cell "fate" or due to the lack of appropriate cell markers their "mixed" state is difficult to be distinguished.

Another example of a "mixed" cell phenotype is the intrinsically photosensitive retinal ganglion cells (ipRGCs) of the mammalian retina that reflect both the characteristics of projection neurons as well as photoreceptors [1, 5, 18, 23]. ipRGCs are able to express the melanopsin photopigment while there are also photosensitive. In this regard, ipRGCs represent a potential example of a mixed/frustrated cell fate. It is speculated that retinal ganglion cells (RGCs) have lost through evolution their photosensitivity characteristic apart from their subfamily of ipRGCs that managed to retain this dual characteristic. It would be rather interesting to test biologically whether Notch activation can seggregate ipRGCs dual function.

Our findings draw also parallels to a recent work by Alfonso Martinez Arias and coworkers [16, 17] that investigated the intermediate transition states that arise during cell fate decision processes as a result of the co-operation of two signaling pathways (Wnt and Notch). This transition state is an emerging highly unstable and reversible state between the initial (pluripotent) and differentiated state.

Lately, Vistulo de Abreu and coworkers [14,15] proposed that frustrated cells can provide another approach of functional immune system. They considered a system of three cells where they try to form stable conjugates. For example, if cells "A" and "B" are conjugated and cell "C" is alone, cell "C" can form a new conjugate with either cell "B" or "A", destroying the previous "AB" conjugate. Therefore, "A" becomes a frustrated cell by the presence of cell "C", and returns to the non-conjugate state. Every conjugate cell can be destabilized by interactions with neighboring cells. It will be rather interesting to investigate the Notch/Delta effect on the aforementioned immune system configuration.

Finally, the existence of transient/frustrated cell phenotype has already been introduced in the epithelial to mesenchymal transition (EMT) [50]. During EMT, cells gain the ability to migrate and invade by loosing epithelial characteristics and gaining mesenchymal attributes. However, between the epithelial (E) and mesenchymal (M) states, there exists an intermediate phenotype known as the partial (P) EMT state. The P state retains both characteristics of epithelial cells as well as features of mesenchymal states. Namely, the $\mathrm{P}$ state represents a transient/frustrated phenotype that it could be transformed to E or M phenotype respectively. Recent experimental and theoretical models revealed that several cell types, starting from the epithelial phenotype, converted to partial EMT and then to mesenchymal phenotypes as concentration of transforming growth factor- $\beta$ (TGF- $\beta$ ) was increased. 
The "frustrated states" identified in our model may also be associated with stochastic fate switching. The effect of noise in stochastic fate switching has already been observed and analyzed in the context of genetic circuits. More specifically, stabilizing and destabilizing roles of noise in genetic circuits have been reviewed in Eldar and Elowitz [2010] (and references therein).

The aforementioned models highlight that transient behavior can be regarded as an evolutionary novel feature of developmental growth.

\section{Conclusions}

The results and ideas discussed above suggest a general framework of the effect of extrinsic noise in the Notch/Delta induced cellular pattern formation. In particular, we have adopted a simplified version of the model described in Collier et al. [1996], which can produce a "salt and pepper" cellular pattern through the use of a Cellular Automaton formulation. We also investigated the impact of the differentiation wave, associated with the Notch/Delta mechanism, on the cell fate specification for the stochastic and deterministic case. In the deterministic case, pattern formation is coordinated by a wave of differentiation. Whereas on the stochastic case extrinsic noise weakens the effect of the differentiation wave. Our analysis showed that even small noise intensities can give rise to "frustrated cell furrows".

However, the observation of "frustrated states" seems to be more an exception than a rule in biological development. Obviously, cells have developed de-noising mechanisms. An example is described in Barad et al. [2010] who identified the existence of error minimization mechanisms associated with the Notch/Delta pathway. Later, Sprinzak et al. [2010] proposed the cis-trans Notch/Delta pathway which includes the exact ingredients for the suppression of stochastic fluctuations. Further design ideas for de-noising mechanisms can be expected from the analysis of extensions of the mathematical model introduced in this paper.

\section{REFERENCES}

[1] D. Arendt, The evolution of cell types in animals: emerging principles from molecular studies, Nat. Rev. Genet. 9 (2008), no. 11, 868-882.

[2] S. Artavanis-Tsakonas, M. D. Rand, and R. J. Lake, Notch signaling: cell fate control and signal integration in development, Science 284 (1999), no. 5415, 770-776.

[3] O. Barad, E. Hornstein, and N. Barkai, Robust selection of sensory organ precursors by the notch-delta pathway, Curr Opin Cell Biol 23 (2011), no. 6, 663-667.

[4] O. Barad, D. Rosin, E. Hornstein, and N. Barkai, Error minimization in lateral inhibition circuits, Sci Signal 3 (2010), no. 129, 1-8.

[5] D. M. Berson, F. A. Dunn, and M. Takao, Phototransduction by retinal ganglion cells that set the circadian clock, Science 295 (2002), no. 5557, 1070-1073.

[6] S. J. Bray, Notch signaling: a simple pathway becomes complex, Nat Rev Mol Cell Bio 7 (2006), no. 9, 678-689.

[7] E. Cau and P. Blader, Notch activity in the nervous system: to switch or not switch?, Neural Dev 4 (2009), no. $36,1-11$.

[8] E. Cau, A. Quillien, and P. Blader, Notch resolves mixed neural identities in the zebrafish epiphysis, Development 135 (2008), no. 14, 2391-2401.

[9] C. L. Cepko, C. P. Austin, X. Yang, M. Alexiades, and D. Ezzeddine, Cell fate determination in the vertebrate retina, Proc Nat Acad. Sci. USA 93 (1996), 589-595.

[10] A. B. Chitnis, The role of notch in lateral inhibition and cell fate specification, Mol. Cel. Neurosci 6 (1995), no. 4, 311-321.

[11] M. Cohen, B. Baum, and M. Miodownik, The importance of structured noise in the generation of self-organizing tissue patterns through contact-mediated cell-cell signalling, J R Soc Interface 8 (2011), no. 59, 787-798.

[12] M. Cohen, M. Georgiou, N. L. Stevenson, M. Miodownik, and B. Baum, Dynamic filopodia transmit intermittent delta-notch signaling to drive pattern refinement during lateral inhibition, Dev Cell 19 (2010), no. 1, 78-89.

[13] J. R. Collier, N. A. M. Monk, P. K. Maini, and J. H. Lewis, Pattern formation by lateral inhibition with feedback: a mathematical model of delta-notch intercellular signalling, J Theor Biol 183 (1996), no. 4, 429-446.

[14] F. V. de Abreu and C. P. Mostardinha, Maximal frustration as an immunological principle, J R Soc Interface 6 (2009), no. 32, 321--334.

[15] F. V. de Abreu, E. N. M. Nolte'Hoen, C. R. Almeida, and D. M. Davis, Cellular frustration: a new conceptual framework for understanding cell-mediated immune responses, Artificial Immune Systems, Springer, 2006, pp. 3751. 
[16] S. M. Descalzo and A. M. Arias, The structure of wntch signalling and the resolution of transition states in development, Semin Cell Dev Biol. 23 (2012), no. 4, 443-449.

[17] S. M. Descalzo, J. de Navascues, and A. M. Arias, Wnt-notch signalling: an integrated mechanism regulating transitions between cell states, Bioessays 34 (2012), no. 2, 110-118.

[18] M. T. H. Do, S. H. Kang, T. Xue, H. Zhong, H. W. Liao, D. E. Bergles, and K. W. Yau, Photon capture and signalling by melanopsin retinal ganglion cells, Nature 457 (2009), no. 7227, 281-287.

[19] A. Eldar and M. B. Elowitz, Functional roles for noise in genetic circuits, Nature 467 (2010), no. 9, $167-173$.

[20] P. Formosa-Jordan, Pattern formation through lateral inhibition mediated by notch signaling, Ph.D. thesis, Facultat de Física, Universitat de Barcelona, 2013.

[21] P. Formosa-Jordan and M. Ibanes, Competition in notch signaling with cis enriches cell fate decisions, PLoS One 9 (2014), no. 4, e95744.

[22] M. E. Fortini, Notch signaling: The core pathway and its posttranslational regulation, Dev Cell 16 (2009), no. 5, 633-647.

[23] S. Hattar, H. W. Liao, M. Takao, D. M. Berson, and K. W. Yau, Melanopsin-containing retinal ganglion cells: architecture, projections, and intrinsic photosensitivity, Science 295 (2002), no. 5557, 1065-1070.

[24] C. P. Hsu, P. H. Lee, C. W. Chang, and C. T. Lee, Constructing quantitative models from qualitative mutant phenotypes: preferences in selecting sensory organ precursors, Bioinformatics 22 (2006), no. 11, 1375-1382.

[25] A. P. Jadhav, S.-H. Cho, and C. L. Cepko, Notch activity permits retinal cells to progress through multiple progenitor states and acquire a stem cell property, Proc Nat Acad. Sci. USA 103 (2006), no. 50, $18998-19003$.

[26] A. P. Jadhav, H. A. Mason, and C. L. Cepko, Notch 1 inhibits photoreceptor production in the developing mammalian retina, Development 133 (2006), no. 5, 913-923.

[27] R. J. J. Johnston and C. Desplan, Stochastic mechanisms of cell fate specification that yield random or robust outcomes, Annual review of cell and developmental biology 26 (2010), 689.

[28] C. T. Kelley, Iterative methods for optimization, vol. 18, Siam, 1999.

[29] Y. Koizumi, Y. Iwasa, and T. Hirashima, Mathematical study of the role of delta/notch lateral inhibition during primary branching of drosophila trachea development, Biophys J 103 (2012), no. 12, 2549-2559.

[30] E. C. Lai, Notch signaling: control of cell communication and cell fate, Development 131 (2004), no. 5, 965-973.

[31] R. Losick and C. Desplan, Stochasticity and cell fate, Science 320 (2008), no. 5872, 65-68.

[32] A. Louvi and S. Artavanis-Tsakonas, Notch signaling in vertebrate neural development, Nat Rev Neurosci 7 (1999), no. 2, 93-102.

[33] G. Maruyama, Continuous markov processes and stochastic equations, Rend. Circ. Mat. Palermo 4 (1955), no. 1, 48-90.

[34] E. Meir, G. Dassow, E. Munro, and G. M. Odell, Robustness, flexibility, and the role of lateral inhibition in the neurogenic network, Curr Biol 12 (2002), no. 10, 778-786.

[35] M. Milan and S. M. Cohen, Notch signaling: Filopodia dynamics confer robustness, Curr Biol 20 (2010), no. 18, R802-4.

[36] M. A. Miodownik, Monte carlo models for grain growth and recrystallisation, ASM handbook series, modelling and simulation: processing of metalilic materials 22 (2009).

[37] M. A. Miodownik, P. Smereka, D. J. Srolovitz, and E. A. Holm, Scaling of dislocation cell structures: diffusion in orientation space, Proc. of the Royal Soc. of London. Series A: Mathematical, Physical and Engineering Sciences 457 (2001), no. 2012, 1807-1819.

[38] A. H. Nayfeh and B. Balachandran, Applied nonlinear dynamics: analytical, computational and experimental methods, 2 ed., John Wiley \& Sons, lnc., 2004.

[39] R. D. O'Dea and J. R. King, Multiscale analysis of pattern formation via intercellular signalling, Math Biosc 231 (2011), no. 2, 172-185.

[40] M. R. Owen, J. A. Sherratt, and H. J. Wearing, Lateral induction by juxtacrine signaling is a new mechanism for pattern formation, Dev. Biol. 217 (2000), no. 1, 54-61.

[41] M. Perron and W. A. Harris, Determination of vertebrate retinal progenitor cell fate by the notch pathway and basic helix-loop-helix transcription factors, Cell. Mol. Life Sci. 57 (2000), no. 2, 215-223.

[42] J. Petrovic, P. Formosa-Jordan, J. Luna-Escalante, G. Abello, M. Ibanes, J. Neves, and F. Giraldez, Liganddependent notch signaling strength orchestrates lateral induction and lateral inhibition in the developing inner ear, Development 141 (2014), no. 11, 2313-2324.

[43] E. Plahte, Pattern formation in discrete cell lattices, J Math Biol 43 (2001), 441-445.

[44] M. Sato, T. Suzuki, and Y. Nakai, Waves of differentiation in the fly visual system, Dev. Biol. 380 (2013), no. 1, $1-11$.

[45] O. Shaya and D. Sprinzak, From notch signaling to fine-grained patterning: modeling meets experiments, Curr Opin Genet Dev 21 (2011), no. 6, 732-739.

[46] J. Shin, J. Poling, H.-C. Park, and B. Appel, Notch signaling regulates neural precursor allocation and binary neuronal fate decisions in zebrafish, Development 134 (2007), no. 10, 1911-1920. 
[47] D. Sprinzak, A. Lakhanpal, L. LeBon, L. A. Santat, M. E. Fontes, G. A. Anderson, J. Garcia-Ojalvo, and M. B. Elowitz, Cis-interactions between notch and delta generate mutually exclusive signalling states, Nature 465 (2010), no. 7294, 86-90.

[48] H. J. Wearing, M. R. Owen, and J. A. Sherratt, Mathematical modelling of juxtarine patterning, Bull. Math. Biol. 62 (2000), no. 2, 293-320.

[49] S. D. Webb and M. R. Owen, Oscillations and patterns in spatially discrete models for developmental intercellular signalling, J Math Biol 48 (2004), no. 4, 444-476.

[50] J. Zhang, X.-J. Tian, H. Zhang, Y. Teng, R. Li, F. Bai, S. Elankumaran, and J. Xing, Tgf- $\beta$-induced epithelialto-mesenchymal transition proceeds through stepwise activation of multiple feedback loops, Sci. Signal. 7 (2014), no. 345 , ra91 1-11. 